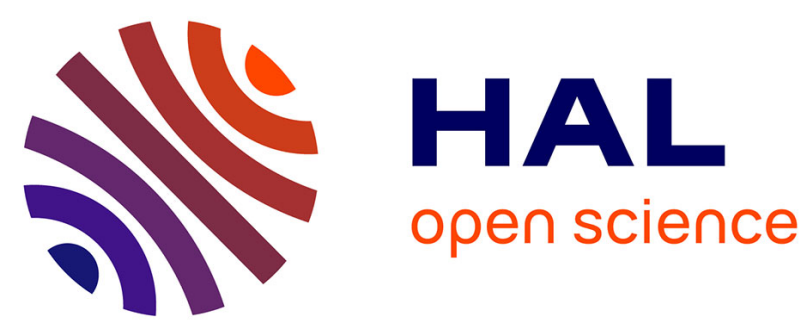

\title{
Collateral effects of targeting the nucleus pulposus via a transpedicular or transannular surgical route: a combined X-ray, MRI, and histological long-term descriptive study in sheep
}

Cyrille Decante, Johann Clouet, Antoine Hamel, Luc Le Fournier, Olivier Gauthier, Dominique M. Rouleau, Julie Lesoeur, Boris Halgand, Catherine Levisage, Jérome Guicheux, et al.

\section{- To cite this version:}

Cyrille Decante, Johann Clouet, Antoine Hamel, Luc Le Fournier, Olivier Gauthier, et al.. Collateral effects of targeting the nucleus pulposus via a transpedicular or transannular surgical route: a combined X-ray, MRI, and histological long-term descriptive study in sheep. European Spine Journal, 2020, Online ahead of print. inserm-02943975

HAL Id: inserm-02943975 https://www.hal.inserm.fr/inserm-02943975

Submitted on 21 Sep 2020

HAL is a multi-disciplinary open access archive for the deposit and dissemination of scientific research documents, whether they are published or not. The documents may come from teaching and research institutions in France or abroad, or from public or private research centers.
L'archive ouverte pluridisciplinaire HAL, est destinée au dépôt et à la diffusion de documents scientifiques de niveau recherche, publiés ou non, émanant des établissements d'enseignement et de recherche français ou étrangers, des laboratoires publics ou privés. 


\section{Query Details}

1. Please check whether the usage of the term 'peroperative' in the caption of Figure ' 1 ' is $\mathrm{OK}$.

\section{Original Article}

\section{Collateral effects of targeting the nucleus pulposus via a transpedicular or transannular surgical route: a combined X-ray, MRI, and histological long-term descriptive study in sheep}

Cyrille Decante, 1,2

Johann Clouet, 1,3,4,5

Antoine Hamel, 1,2

Luc Le Fournier, 6

Olivier Gauthier, 1,7

Dominique Rouleau, 1,8

Julie Lesoeur, 1,3

Boris Halgand, 1,3,9

Catherine Le Visage, 1

Jérôme Guicheux, $1 \bowtie, 3,10$

Email jerome.guicheux@inserm.fr

Marion Fusellier, 1,8

1 INSERM UMR 1229, RMeS, Regenerative Medicine and Skeleton, School of Dental Surgery, ONIRIS, Université de Nantes, 1-Place Alexis 
Ricordeau, 44042 Nantes Cedex I, France

2 CHU Nantes, PHU5 HME Chirurgie Infantile, 44093 Nantes, France

${ }^{3}$ UFR Odontologie, Université de Nantes, 44042 Nantes, France

${ }^{4}$ UFR Sciences Biologiques et Pharmaceutiques, Université de Nantes, 44035 Nantes, France

5 CHU Nantes, Pharmacie Centrale, PHU11, 44093 Nantes, France

6 Department of Neurosurgery, CHU Angers, 49033 Angers, France

7 Department of Surgery,CRIP, Oniris College of Veterinary Medicine, Food Science and Engineering, 44307 Nantes, France

8 Department of Diagnostic Imaging, CRIP, Oniris College of Veterinary Medicine, Food Science and Engineering, 44307 Nantes, France

9 CHU Nantes, 44093 Nantes, France

10 CHU Nantes, PHU4 OTONN, 44093 Nantes, France

Received: 13 September 2019 / Accepted: 7 September 2020

\section{Abstract}

\section{Purpose}

In the context of regenerative medicine strategies, based in particular on the injection of regenerative cells, biological factors, or biomaterials into the nucleus pulposus (NP), two main routes are used: the transpedicular approach (TPA) and the transannular approach (TAA). The purpose of our study was to compare the long-term consequences of the TPA and the TAA on intervertebral disc (IVD) health through a longitudinal follow-up in an ovine model.

\section{Methods}

The TPA and the TAA were performed on 12 IVDs from 3 sheep. Six discs were left untreated and used as controls. The route and injection feasibility, as well as the IVD environment integrity, were assessed by MRI (T2-weighted signal intensity), micro-CT scan, and histological analyses (Boos' scoring). The sheep were assessed at 1,3, and 7 months. 


\section{Results}

Both the TPA and the TAA allowed access to the NP. They both induced NP degeneration, as evidenced by a decrease in the T2wsi and an increase in the Boos' scores. The TPA led to persistent end-plate defects and herniation of NP tissue (Schmorl's node-like) after 7 months as well as the presence of osseous fragments in the NP.

\section{Conclusions}

The TPA induced more severe lesions in IVDs and vertebrae compared to the TAA. The lesions induced by the TPA are reason to consider whether or not this route is optimal for studying IVD regenerative medicine approaches.

\section{Keywords}

Intervertebral disc degeneration

Transpedicular approach

Transannular approach

Regenerative medicine

Cyrille Decante, Johann Clouet: Co-first author

\section{Introduction}

Low back pain (LBP) is an extremely frequent symptom and in $40 \%$ of cases it is associated with intervertebral disc (IVD) degenerative disease (DDD) $[1,2,3]$. Current therapies for LBP mainly manage the pain experienced by patients using pharmacological treatments that include NSAIDs and analgesics. Treatment of the most advanced cases of LBP typically involves surgery (e.g. spine fusion and total disc replacement). Despite significant technical improvements, these surgical procedures remain invasive. Spine fusion and total disc replacement have been reported to have similar rates of complications, while a lower rate of adjacent segment degeneration has been observed with arthroplasty [4].

The improvement of our knowledge regarding IVD degeneration has recently fostered the development of cell-based regenerative medicine as an etiopathogenic option for treatment of DDD [5, 6]. Adding mesenchymal stem cells (MSC) that exhibit a broad range of biological properties (antiinflammatory, anti-catabolic, and pro-regenerative) to the NP is of particular interest. An increasing body of preclinical and clinical evidence has been 
generated in support of the notion that intradiscal injection of MSC may be a clinically translatable option for managing DDD $[7,8,9,10]$.

To demonstrate the preclinical relevance of such cell-based therapies, several DDD animal models have been described, involving mice, rabbits, pigs, and sheep [11]. However, no particular animal model stands out among the various models that have been devised to date. The ovine model exhibits spontaneous DDD with a physiopathology that is quite similar to that of human DDD [11, 12]. Moreover, there are a number of anatomical and biomechanical similarities between human and sheep IVDs and vertebrae [13, 14].

A key aspect that affects the efficacy of IVD cell-based therapies is in regard to the choice of the surgical approach to safely inject the cell-based therapeutic product into the NP while also limiting the risk of exacerbating the DDD. The current "gold standard" for targeting the NP is the percutaneous dorsolateral surgical route involving needle puncture of the annulus fibrosus (AF), referred to as the transannular approach (TAA) $[15,16]$. However, it is now well recognized that the TAA can lead to in-depth alteration of the mechanical properties of the AF. Such AF impairments are known to lead to an increased risk of NP herniation and accelerated IVD degeneration [17, 18, 19, 20, 21], as has also been described in human IVDs following AF puncture-mediated discography [22]. In addition, it should also be stressed that puncture of the AF, with a critically sized diameter needle, is a widely used animal model of surgically induced DDD $[17,23,24]$. In the context of IVD regenerative medicine, it is, therefore, essential to consider alternative approaches than the TAA. Vadalà et al. have described a promising transpedicular approach (TPA) [25] that consists of reaching the NP through the caudal vertebra by successively crossing the pedicle, the vertebral body, and the endplate, thereby protecting the AF from lesions. Despite encouraging results [26] related to the surgical feasibility of this route, using an ex vivo ovine model, we recently reported that the TPA can induce the migration of intradiscal osseous fragments or endplate fracture [27], as evidenced by micro-computed tomography and histological analyses. However, this preliminary ex vivo study did not take into account the complex in vivo mechanical environment of IVDs and it did not address the long-term in vivo effects after the TPA. To address both of these clinically relevant issues, the aim of the present study was to compare the long-term consequences of the TPA and the TAA on IVDs and vertebral endplate integrity through a longitudinal followup in an ovine model.

\section{Materials and Methods}

\section{Ethical Aspects, animals, and treatment conditions}


All of the animal handling and surgical procedures were approved by the French Ministry of Agriculture and by the ethics committee of the Pays de la Loire Region (Ethics approval number APAFIS: 8401); and they were performed in the accredited Centre of Research and Pre-clinical Investigations (CRIP) at the ONIRIS-National Veterinary School of Nantes. Three young healthy sheep (Vendée breed, 30-40 kg, 8-10 months, GAEC HEAS farm, Ligné F-44850) were used. Six lumbar IVDs (T13-L1, L1-L2, L2-L3, L3-L4, L4-L5, and L5-L6) per animal were used for the experiments $(n=18)$. For each sheep, four IVDs were randomly treated (Table 1): two IVDs were accessed by the transpedicular approach (TPA) and two were accessed by the transannular approach (TAA). Two IVDs were not treated and hence used as controls (CTL). The housing conditions were the same for the three sheep. To evaluate the feasibility of injection into the NP, iodine contrast agent (iCA) was randomly injected for each sheep in one IVD treated by TPA and one IVD treated by TAA. The iCA was ioxaglic acid (Hexabrix ${ }^{\circledR} 320 \mathrm{mg} / \mathrm{ml}$, Guerbet, France) diluted to $10 \%$ in a physiological saline solute. After the surgery, the sheep were followed for 1, 3, or 7 months before being euthanized to remove the lumbar spine. One additional sheep was used as a day 0 reference for the histological analyses.

\section{Table 1}

Summary of the surgical procedures performed on the sheep IVDs. The mention « iCa» indicates iodine contrast agent was injected on IVD (CTL: no treatment, TAA: transannular approach, TPA: transpedicular approach)

\begin{tabular}{|l|l|l|l|}
\hline $\begin{array}{l}\text { Disc } \\
\text { levels }\end{array}$ & $\begin{array}{l}\text { Sheep } 1 \text { follow-up } \\
\text { 1 month }\end{array}$ & $\begin{array}{l}\text { Sheep } 2 \text { follow-up } \\
\text { months }\end{array}$ & $\begin{array}{l}\text { Sheep } 3 \text { follow-up } \\
\text { months }\end{array}$ \\
\hline T13-L1 & CTL & TAA (iCa) & TPA \\
\hline L1-L2 & TPA (iCa) & CTL & TAA \\
\hline L2-L3 & TAA & TPA (iCa) & CTL \\
\hline L3-L4 & CTL & TPA & TPA (iCa) \\
\hline L4-L5 & TPA & CTL & TAA (iCa) \\
\hline L5-L6 & TAA (iCa) & TAA & CTL \\
\hline
\end{tabular}

\section{Surgical procedure}

The surgical procedure was performed under general anaesthesia (induction: diazepam $0.2 \mathrm{mg} / \mathrm{kg} \mathrm{IV}$, ketamine 2 to $5 \mathrm{mg} / \mathrm{kg} \mathrm{IV}$, propofol 1 to $3 \mathrm{mg} / \mathrm{kg}$, then placed under gaseous anaesthesia NO: $2 \mathrm{~L} / \mathrm{min}, \mathrm{O}_{2}: 2 \mathrm{~L} / \mathrm{min}$, isoflurane: $2 \%$ ). The sheep were placed in a ventral decubitus position. The entire surgical procedure was performed under fluoroscopic guidance (OEC9800plus ${ }^{\circledR}$, General 
Electric GE Healthcare, Little Chalfont, UK). For the TPA condition, the percutaneous technique was adapted from the surgical approach described by Vadalà et al. [25] and Le Fournier et al. [27]. It was carried out using a 2-mm Jamshidi ${ }^{\circledR}$ (ADHESIA MEDICAL, Flaxlanden, France) bone marrow biopsy needle employing a hollow trocar. When the trocar was under the endplate, a threaded 1.5-mm Kirschner wire was used to slowly cross the endplate to the NP through the cannula with a drill under video-fluoroscopic guidance. The iCA was injected with a spinal needle (Mila Spinal Needle ${ }^{\circledR} 20 \mathrm{G}, 20 \mathrm{~cm}$, Mila International Inc, Florence, KY, USA). Unlike in the Vadalà et al. protocol, no endplate repair was performed. For the TAA condition, a transannular approach was performed percutaneously, as in the classic posterolateral procedure, with a $22 \mathrm{G}$ needle (Spinocan ${ }^{\circledR} 22 \mathrm{G}, 88 \mathrm{~mm}, \mathrm{~B}$ Braun, Berlin, Germany). The iCA was injected using this spinal needle. For both of the conditions, the amount of iCA injected was $100 \mu \mathrm{L}$.

\section{MRI procedure follow-up}

MRI of the entire lumbar spine was performed under anaesthesia as described for the surgical procedure. It was carried out before the surgery on each sheep to exclude spontaneously degenerated IVD or major anatomical abnormalities such as spinal malformations. The images were used as a reference for the in-vivo follow-up. MRI was performed immediately after the surgery, then once a month until the sheep were euthanized, in order to assess the change in the NP signal. The last MRI was performed immediately before the euthanasia. A 1.5T MRI scanner (Magnetom Essenza ${ }^{\circledR}$, Siemens Medical Solutions, Erlangen, Germany) with a standard spine coil was used to obtain T2-weighted images in all three planes (TR: $3000 \mathrm{~ms}$; TE: $86 \mathrm{~ms}$ ) with the following parameters: matrix: $512 \times$ 358 ; field of view $350 \times 350 \mathrm{~mm}$; and slice thickness: $3 \mathrm{~mm}$ with no interslice gap.

\section{Computed tomography scan (CT scan) procedure}

The three sheep were euthanized at 1,3, or 7 months (sedation: ketamine $5 \mathrm{mg} / \mathrm{kg}$, then euthanasia: intravenous injection of a sodium pentobarbital overdose). The entire lumbar spine was harvested and isolated as an "endplateIVD-vertebra" segment by cutting the vertebrae transversely with a band saw. The caudal vertebral body was sectioned below its transverse process, just above the caudal endplate, in order to preserve the canal of the TPA. To observe the effects of the TPA on the vertebrae, the endplates crossed by the drill and the IVDs were analysed by CT scanning (Inveon ${ }^{\circledR}$, Siemens Medical Solutions, Erlangen, Germany) with the following parameters: low magnification, time of exposure $550 \mathrm{~ms}, 80 \mathrm{kV}, 500 \mu \mathrm{A}$, and slice thickness $59 \mu \mathrm{m}$. 


\section{Image analysis}

The images were analysed with Osirix ${ }^{\circledR}$ software (Osirix Foundation, Geneva, Switzerland). For the MRI images, the T2-weighted signal intensity (T2wsi) was determined on MRI images of a midsagittal slice. The T2wsi was determined by the ratio of the NP weighted mean signal intensity divided by the spinal cord signal, and it was compared with the preoperative images. The CT scan images were also analysed with Osirix ${ }^{\circledR}$ software. The volume of the osseous fragments in the IVDs was calculated after defining the region of interest and it was expressed in $\mathrm{mm}^{3}$.

\section{Histological analyses}

The "endplate-IVD-vertebra" segments were obtained by sawing the vertebral bodies immediately above the cranial endplate and just beneath the caudal endplate. The endplates were then sanded to reduce the bone thickness (Metaserv ${ }^{\circledR} 2000,800$, Buehler, Switzerland). Segments were fixed in 4\% paraformaldehyde, decalcified in TBD-2 ${ }^{\circledR}$ decalcifier solution for twelve days (Thermo Fisher Scientific, Waltham, MA, USA), frozen and then first sectioned transversely, taking care to preserve the caudal endplate. Secondly, the IVD with the caudal endplate was sectioned sagittaly to observe the route of the TPA and the endplate disruption. Sections of $10 \mu \mathrm{m}$ were generated with a cryostat (CryoStar NX70®, Thermo Fisher Scientific, Waltham, MA, USA). The sections were stained with haematoxylin eosin (HE) and $0.1 \%$ Alcian blue (SigmaAldrich, St. Louis, MO, USA) as previously described [28]. Masson's trichrome staining was carried out on the sagittal segments to assess the effects of the TPA on the endplates. The histological transversely sections were also analysed using a modification of Boos' scoring [29]. Briefly, this Boos scoring was based on the analysis of four criteria regarding the integrity of the Nucleus pulposus: a decrease in cell density, granular changes, tear and cleft formation (except for the cleft induced by needle puncture of the endplate), and mucus degeneration. Some of the parameters were ranked from 0 to 4 (granular and mucus degeneration) and others were ranked from 0 to 5 (a decrease in cell density, and tear and cleft formation) depending on the intensity of the parameters tested ( 0 , lowest; 4 or 5 , highest). The stained sections were observed with a slide scanner and all of the sections were analysed with NDP view ${ }^{\circledR}$ software (Hamamatsu, Japan). The day 0 Boos' scores were obtained from an additional sheep who was euthanized immediately after the surgery. Three independent investigators who had expertise with reading histological slides performed a blind evaluation of the histological samples. The reported results are the means of the scores from each investigator.

\section{Results}




\section{Route and injection feasibility}

Six TPA, as well as six TAA, were carried out in this study (two TPA and two TAA on each sheep). In terms of the accuracy of the procedure, all (100\%) of the TPA and the TAA were successful based on the fluoroscopic guidance and the postoperative MRI data (Fig. 1a, b). No pedicle fracture or encroachment into the spinal canal was observed. Injection into the NP by the TPA was feasible, as confirmed by the fluoroscopic guidance. One case of iCA backflow was observed (Fig. 1c), localized in the canal of the TPA and inside the vertebra. No iCA backflow was observed after the TAA.

\section{Analysis of IVD integrity}

\section{CT scan analysis}

The IVDs and lumbar vertebrae were analysed by CT scanning at 1, 3, and 7 months in order to study bone lesions induced by the TPA and the TAA. No false route in the spinal canal was observed with the TPA. No pedicular fracture sequelae were observed. The endplates had been crossed without inducing secondary endplate fractures.

At 7 months, the path in the vertebrae induced by the TPA was still visible. Osteocondensation around the canal and the initiation of bone filling were observed but the bone healing was incomplete. However, no bone filling was observed at the level of the endplate defect. Osseous fragments were found in all of the IVDs treated by the TPA (Fig. 2). These fragments were larger on the IVDs at the 7-month follow-up (mean $2.32 \mathrm{~mm}^{3}$ ), compared to fragments at the 3 - and the 1-month follow-up (mean $0.59 \mathrm{~mm} 3$ and $0.27 \mathrm{~mm}^{3}$, respectively). No osseous fragments were found in the control IVDs or in the IVDs treated by the TAA.

\section{Fig. 1}

Representative images are shown preoperative (a), postoperative (b) sagittal MRI and peroperative dorsoventral fluoroscopy (c) during TPA of the L2-L3 disc level. The path of the TPA (red arrow on (b)) through the endplate to the NP confirmed the TPA feasibility in ovine model. Peroperative dorsoventral fluoroscopy (c) showed one case of iCA reflux through the path of the TPA (blue arrow). T2wsi was subsequently determined as described in Materials and Methods (green circles). (NP: Nucleus pulposus, iCA: iodine contrast agent, TPA: transpedicular approach). Scale bar: $25 \mathrm{~mm}$. 

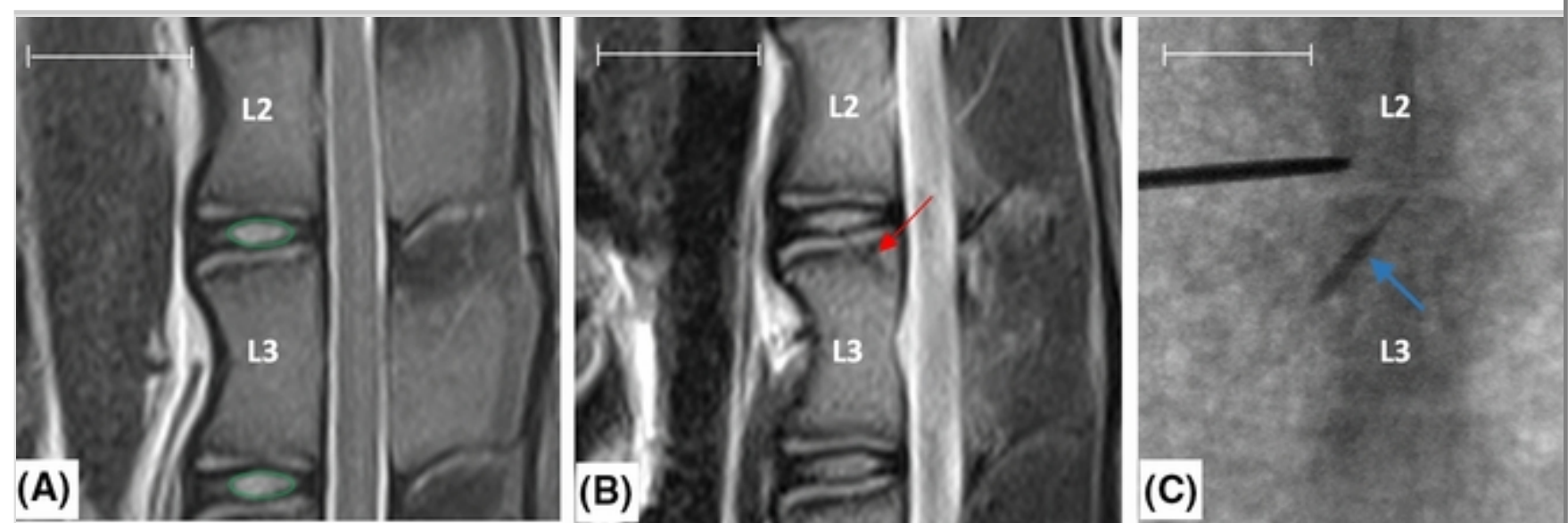

AQ1

Histological analysis

To determine whether the TPA can result in endplate lesions, we performed histological analyses at 1,2, and 7 months. The TPA inevitably causes endplate lesions. At 7 months, the endplate lesions induced by the TPA were not cicatrized and the endplate defects were filled by tissue that was similar to the NP in appearance (Fig. 3). Indeed, NP extravasation through the caudal vertebral body was observed. It was comparable to an intrabody disc herniation analogous to a Schmorl node (Fig. 3). The diameter of these disc herniations was, on average, $1.1 \mathrm{~mm}$. As expected, no endplate lesions were found in the control or in the TAA conditions.

\section{Fig. 2}

CT scan reconstructions of IVDs and caudal vertebrae. Representative pictures of $3 \mathrm{D}$ analysis reconstructions. The volumes of the osseous fragments in the IVDs were determined as described in the Materials and Methods section. No osseous ectopic fragments were observed in the IVDs treated by the TAA or in the control IVDs. The volumes of the osseous ectopic fragments were determined (the values are indicated on the images). (CTL: control IVDs, TPA: transpedicular approach, TAA: transannular approach). Scale bar: $5 \mathrm{~mm}$ 


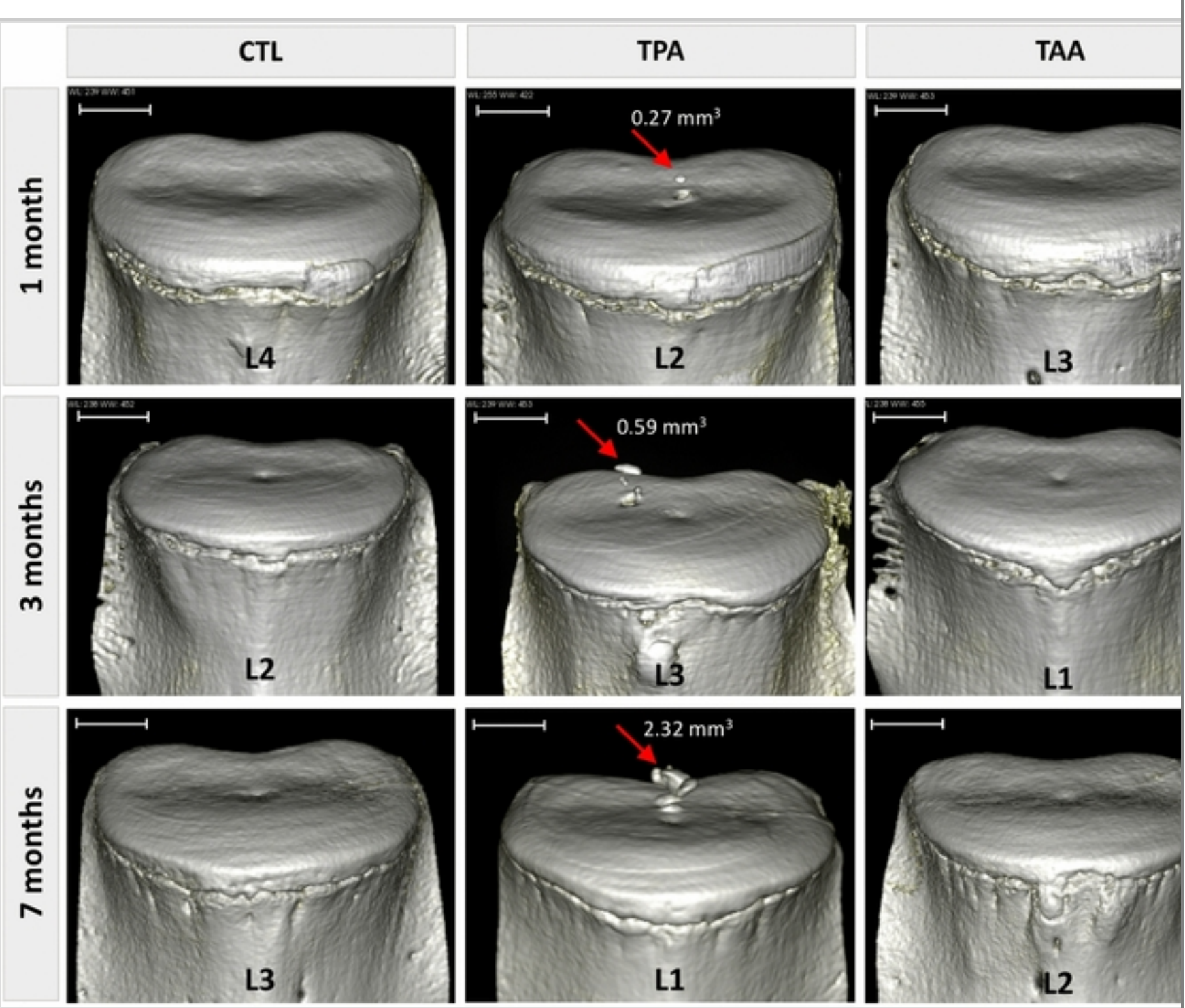

Degenerative effect of the TPA and the TAA

MRI T2-weighted signal intensity (T2wsi)

To evaluate the impact of the TPA and the TAA on the NP degenerative status, MRI was performed and the T2wsi was measured once a month until euthanasia. For the control IVDs, the T2wsi images revealed a spontaneous decrease at 7 months (33.5\% and 19.7\%) (Fig. 4). Although a statistical analysis was not possible due to the limited number of animals, for the IVDs treated by the TPA, the decrease in the T2wsi tended to be more pronounced than that observed in the control IVDs, or in the IVDs treated by the TAA $(47.5 \%$ and $33.3 \%$ for the TPA; $34.1 \%$ and $26.3 \%$ for the TAA). Regardless of the reach, no obvious effect of iCa injection was observed on IVDs at 7 months.

Fig. 3

Representative section are shown IVD, endplates and vertebrae integrity after control, TPA and TAA surgical approaches. Masson's trichrome staining was performed as described in Materials and Methods. Endplate lesions were observed 
after TPA conditions with an absence of healing after seven months. This endplate defects were filled by NP-like tissue similar to a Schmorl node (Red Arrow). No endplate lesion was observed for CTL and TAA conditions. (IVD: intervertebral disc, EP: endplate, V: vertebra). Scale bar: $1 \mathrm{~mm}$
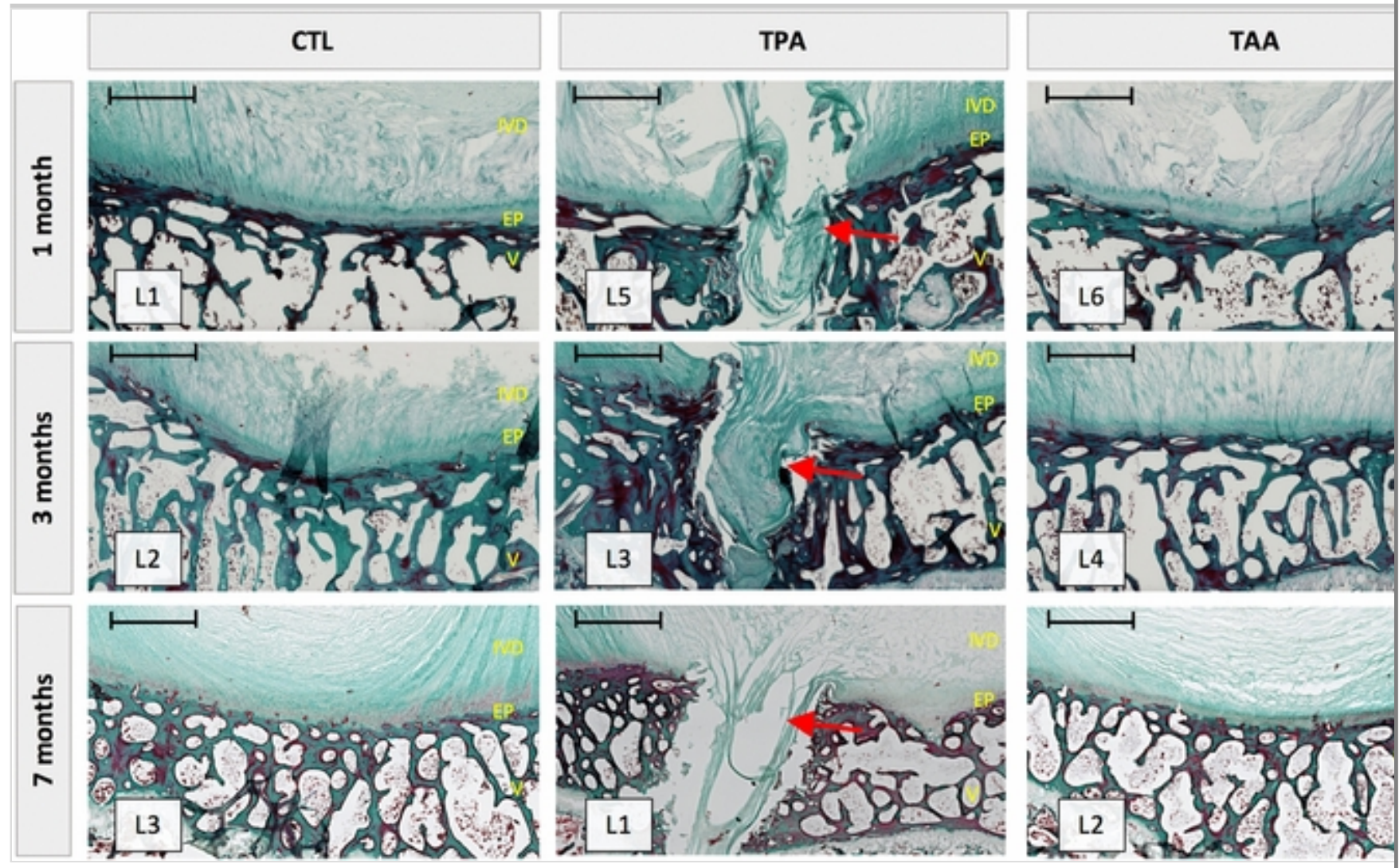

Boos' scoring

To further determine whether the different surgical routes can induce IVD degeneration, Boos' scoring was performed on all the IVDs (Fig. 5). Compared to the control condition, the IVDs treated by the TPA and the TAA exhibited a progressive increase in Boos' scores from day 0 (6, 7 and 6 , respectively) to 7 months (7, 13 and 11, respectively). Although statistical analysis was not possible due to the limited number of samples per group, this increase appeared to be more pronounced at each time point after the TPA compared to the TAA. In the same way as above, no effect of iCA injection was observed on IVDs at 7 months.

Fig. 4

Representative graph is shown the decrease in T2wsi after surgery. Lumbar IVDs of sheep were treated according to either the CTL, TAA and TPA conditions and MRI analysis of T2wsi of sheep lumbar were performed at the indicated times as described in Materials and Methods. For IVDs treated by TPA, the decrease in T2wsi tended to be more pronounced than those observed in control IVDs or IVDs 
treated by TAA. (IVD: intervertebral disc, CTL: control, TPA: transpedicular approach, TAA: transannular approach)

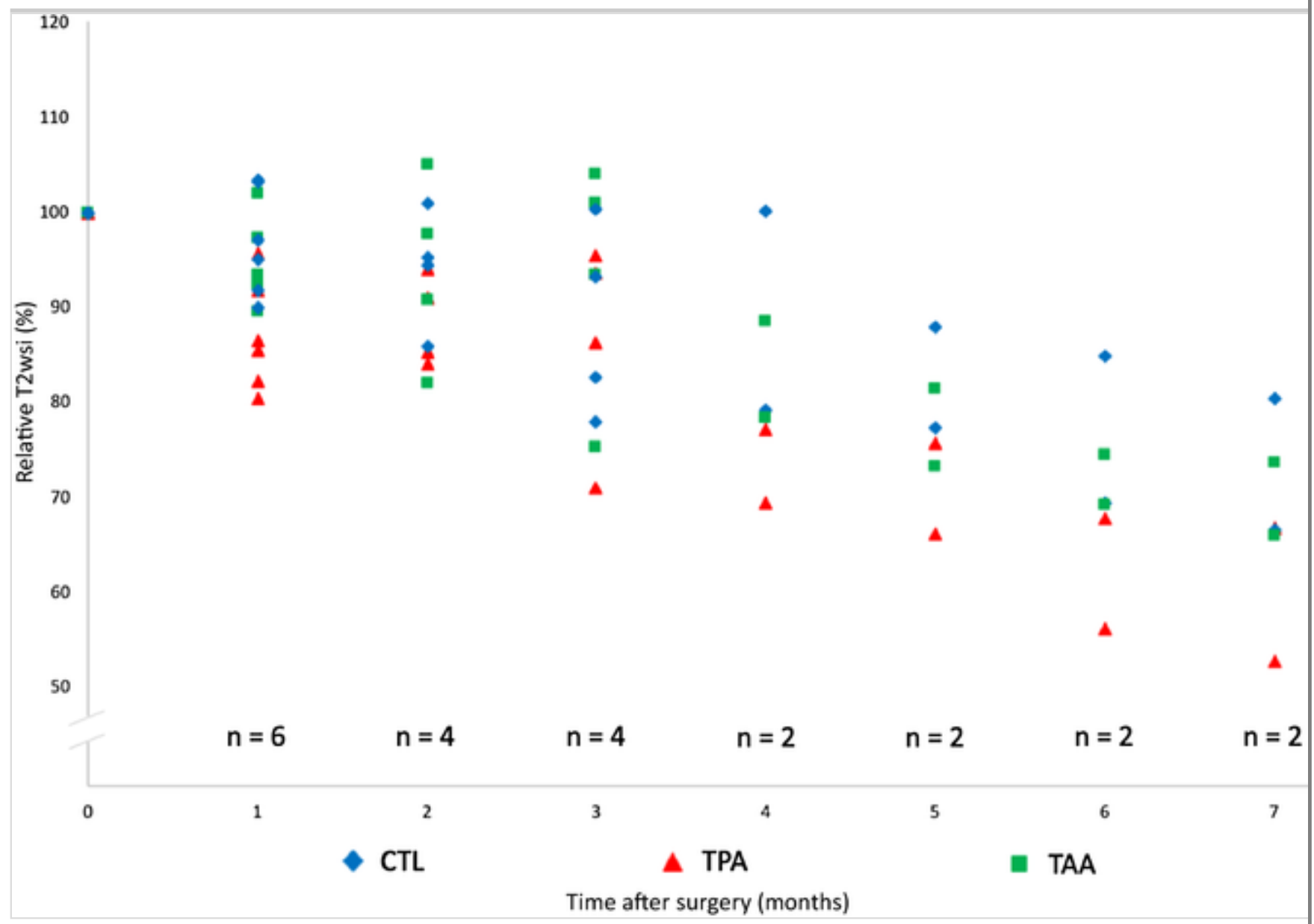

Fig. 5

A representative graph is shown of the IVD degeneration impact of the TPA and the TAA on the Boos' scores and the NP cell density. Lumbar IVDs of sheep were treated according to either the CTL, TAA, or TPA conditions as described in the Materials and Methods section. The Boos' scores were determined for the different conditions after the indicated times as described in the Materials and Methods section. (IVD: intervertebral disc, CTL: control, TPA: transpedicular approach, TAA: transannular approach) 


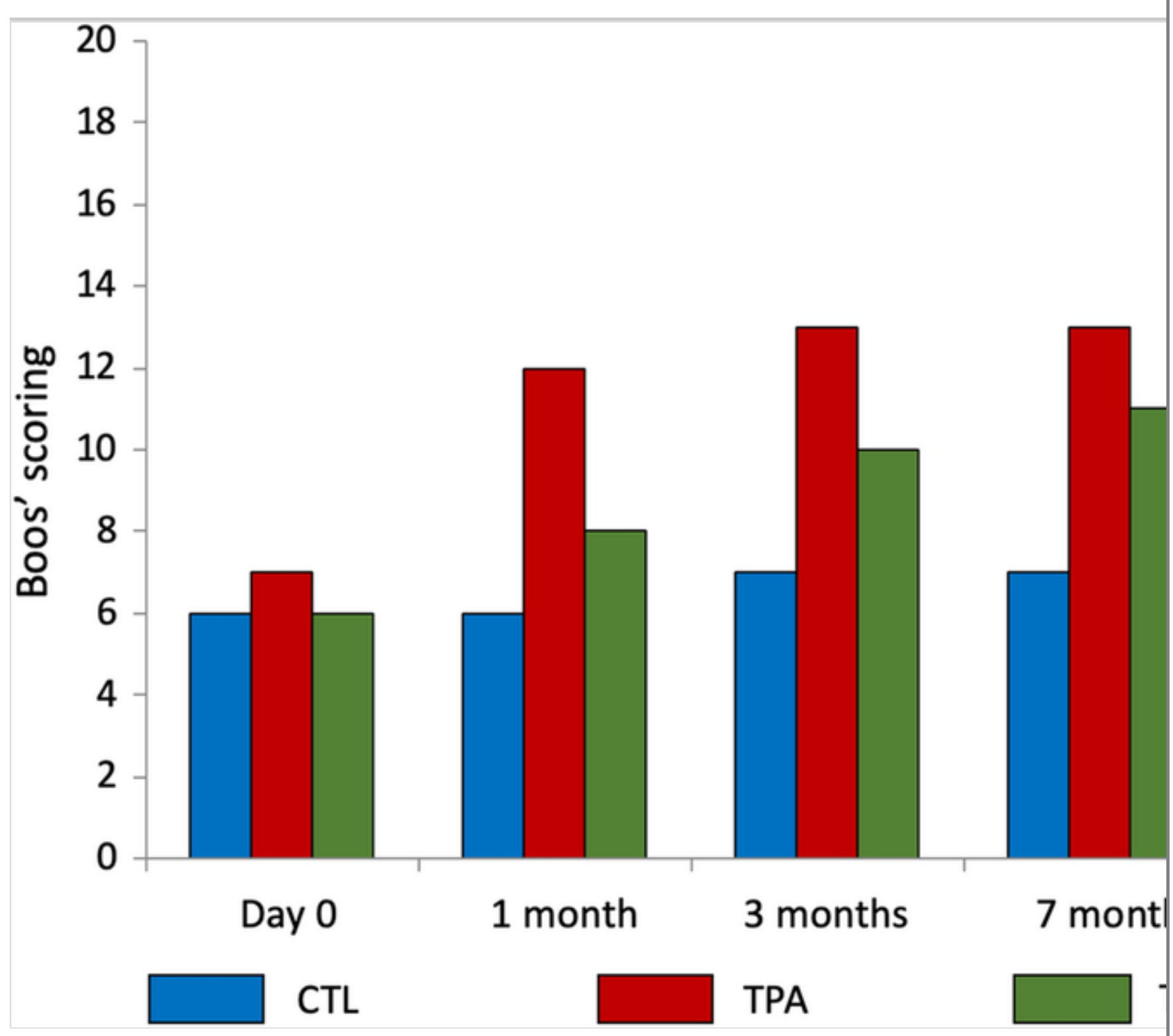

\section{Discussion}

Understanding of the IVD degenerative process has allowed innovative DDD therapies to be developed, notably cell-based therapies based on the injection of cells or biological factors into the NP. Access to the NP needs to be minimally invasive to preclude adverse impacts on the IVD, and the TPA could be an alternative approach to avoid compromising the integrity of the AF. Vadalà et al. were the first to describe the TPA, demonstrating its feasibility in an ovine model [25], with very low peri- and post-operative complications in an in vivo study [26]. To further assess whether the TPA may be a valuable route to target the NP in the general context of IVD regenerative medicine, we performed an in vivo study with a long-term longitudinal follow-up that involved comparison of the TPA with the TAA.

To develop these innovative therapies, and before transposing the NP approach to humans, animal models of DDD that closely mimic the spontaneous IVD 
degeneration observed in humans are required. First, the physical dimensions should allow safe surgical targeting of the NP, as is the case in humans.

Secondly, the pathogenesis and the biomechanical properties should be similar to those of human IVDs. Thirdly, the model should be readily available, and the animal agistment should be as straightforward and inexpensive as possible. Unfortunately, none of the available models generated to date exhibit all of the required features. As already mentioned, the ovine model has biomechanical similarities, and it exhibits spontaneous DDD that is quite similar to that of human DDD at the cellular level $[12,14]$. Moreover, the size of sheep allows surgical techniques to be used that can be transposed to humans. Comparable models are available, and the porcine model has already been used in numerous studies in the context of IVD regenerative medicine [30, 31]. The anatomical features of lumbar spine and IVDs are similar between pigs and humans [32], especially in terms of the size of the pedicles, which is a very relevant aspect for studying the TPA. Moreover, the biomechanical similarities are now wellaccepted [33]. However, in pigs, notochordal cells persist in the NP, even after skeletal maturity has been reached [34], which contrasts with the situation in humans and sheep. In light of the pivotal role of the loss of notochordal cells in IVD degeneration [35], it seems reasonable to speculate that sheep, unlike pig, is a relevant preclinical model for assessing cell-based IVD regenerative medicines.

As previously described [25, 27], and confirmed in our study, the TPA allows access to the NP. Unlike a recent study performed by our group [27], no evidence of pedicle fracture or encroachment into the spinal canal was noted in our study. Among the three discographies performed by the TPA, one case of leakage of the iCA through the transpedicular canal in the vertebra was observed. This leakage and backflow may be explained by the high swelling pressure observed in healthy IVDs, particularly in the ovine model [36]. Further experimentation is needed to confirm this hypothesis. Moreover, degenerated and dehydrated IVD may have a lower swelling pressure [37], although the NP of a degenerated IVD is more fibrous [35] and injection into the NP can also be difficult. Leakage of intradiscal injected substitute, particularly when injecting a cell therapy product, is a major concern, as it has been shown in rabbits that these leaks can lead to osteophyte formation [38].

In addition, the TPA can give rise to venous embolisms, as previously described [27], due to the high swelling pressure of the NP. Other bone lesions after the TPA not found in our study but previously described, such as a pedicle fracture or a false passage, can induce biomechanical disorders and spondylarthritis [26, 27]. These can lead to medullar lesion or neurological impairment. 
Contrarily to Le Fournier et al. [27], no endplate fracture occurred, probably because a drill was used for the procedure to access the NP through the endplate. However, osseous fragments were observed in all of the NP treated by the TPA, as also described in Le Fournier et al. [27]. Intuitively, these fragments may have been carried directly into the IVD by the TPA. Moreover, these fragments could be the result of intradiscal calcification secondary to an inflammatory process, especially as these osseous fragments appeared larger at 7 months compared to at 1 or at 3 months. Indeed, the TPA may induce intradiscal osteogenesis when blood and microscopic bone fragments from the vertebral body remain in the IVD. This would, theoretically, increase the risk of vertebral fusion, thereby amounting to a failure of the IVD regenerative medicine approach. A previous in vivo longitudinal follow-up study of the TPA in an ovine model did not confirm this hypothesis [26]. Nevertheless, the nucleotomies that were performed in this study could have aspirated bone fragments, thereby leading to an underestimation of the risk of intradiscal osteogenesis.

The histological and CT analyses did not show healing of the endplates at 7 months after the TPA, thus confirming data recently obtained by Vadalà et al. [39]. Interestingly, endplate defects were filled by tissue that was similar to the NP in appearance. The high swelling pressure of the NP could explain its extravasation through the endplate, which could then prevent it from healing [36], according to the biological inhibitory effects of IVD cells on osteoblasts, as recently described [40]. The critical endplate defect induced by the TPA is a major limitation of this approach because endplates are considered to be pivotal structures for maintaining IVD homeostasis, especially for providing nutritive support to IVDs and for elimination of waste products. Lesions or sclerosis of the endplate decrease the diffusion-mediated exchange between endplates and IVDs [41] and they accelerate IVD degeneration [42]. Moreover, endplate defects have been shown to initiate the cascade of events that leads to DDD [43]. A height intradiscal pressure is need for fluid and metabolite exchange and the decrease in IVD pressure reduced theses exchanges, as it is already demonstrated in particular in post-traumatic disc degeneration [44]. Vadalà et al. repaired the endplate after the TPA by sealing the edge of the tunnel using a press-fit porous polyurethane cylinder [25, 39]. However, filling the TPA tunnel does not solve the problem of reflux during the procedure. Furthermore, even when the scaffolds were colonized by cells, the histological analyses by Vadalà et al. indicated that the endplates were not fully repaired [39].

Our study showed that there was a deleterious effect of the TPA on the IVD, with an increase in the Boos' scores. Based on the T2wsi longitudinal follow-up, the NP tended to undergo a degree of degeneration. Indeed, the decrease in the 
T2wsi was greater when the IVDs were treated by the TPA as compared to the control. This decrease reflects NP dehydration, which represents a first step in the initiation of the IVD degenerative process. Although our observations could not be proven by statistical analysis due to the limited number of animals, they are in agreement with those of Cinotti et al. [45], who described a porcine model of DDD induced by endplate lesion that was carried out as in our study using a 1.5-mm Kirschner wire. Our results are also in agreement with those of Vadalà et al., who described a stepwise ovine model of IVD degeneration with intact AF induced by an endplate lesion [39]. Although MRI is nowadays among the best in vivo investigative methods to detect DDD, T2-weighted images are not the most sensitive sequence. The use of the MR relaxation time (T2 and T2* mapping) could be more sensitive for detection of early IVD degeneration [46]. Thus, T2 mapping should be used later on to follow induced IVD lesions.

The results of our longitudinal follow-up (lack of healing of the endplate, intradiscal osseous fragments, T2wsi decrease, Boos' score increase) indicate that care must be taken when choosing an approach to the NP in the context of regenerative medicine. Due to the risk of osseous fragments in the NP, we consider it preferable to associate the TPA with a nucleotomy in the context of cell therapy involving injection of exogenous cells and growth factors. Lesions of the AF by large-sized material necessary for nucleotomy by the TAA could be avoided with this approach. However, nucleotomy is not desirable in the context of endogenous repair, as the injection of biological factors must stimulate local endogenous cells and it could be deleterious to IVD resident regenerative cells [47]. In this context, the TAA appears to be more appropriate. Currently, the TPA would be too intrusive and it would carry the risk of early DDD induction and loss of the injected factors by leakage. Although the TAA is controversial [17, 22], a study has shown that the risk of DDD induction after injection through the $\mathrm{AF}$ is not constant, as it depends on the ratio between the gauge of the needle and the height of the IVD [23]. According to Elliott et al., when the gauge of the needle is less than $40 \%$ of the height of the IVD, AF puncture with this needle does not induce accelerated DDD [23]. Carragee et al. [22], used 22 and 25 gauge needles, corresponding to less than $10 \%$ of the height of the IVD, thus suggesting that, in this case, the AF puncture was not responsible for the DDD. While there is a discrepancy in needle size which may have contributed to increased degeneration in TPA with the larger needle size [23], the principal aim of this work was to compare two currently used routes to access the NP. We have decided to use the NP approaches as they have been described in detail and they are used in the literature [22, 23, 25, 27]: the TAA generally entails use of a needle of approximately $0.7 \mathrm{~mm}$ in ovine IVDs, and the TPA involves use of a needle of approximately between 1.5 and $2 \mathrm{~mm}$. In the context of the development of IVD regenerative medicine strategies, it is widely acknowledged 
that the structural integrity of the AF needs to be preserved. Use of a large-size needle $(1.5 \mathrm{~mm})$, in addition to making the TAA surgically more difficult due to the limited height of the IVD, would most likely have resulted in damage to the annulus fibrosus, especially since on a number of occasions we have noticed that lumbar IVDs of sheep (when less than 1 year old) can be very thin (between 2 and $3.5 \mathrm{~mm}$ ), as indicated by our X-ray, MRI, and histological data (data not shown). In this context, using a $1.5 \mathrm{~mm}$ needle to perform the TAA would most likely have led to IVD degeneration. Regarding the needle used for TPA, it remains technically unfeasible to perform the TPA with a $0.7 \mathrm{~mm}$ needle, since such a needle with a sufficient length and mechanical resistance to penetrate through the skin and endplate to the NP is not commercially available.

As the optimal route for targeting the NP in regenerative medicine has yet to be established, other strategies based on homing of endogenous progenitor cells to the diseased tissue warrant further consideration. A number of targeted systemic treatments could be specifically designed to mobilize and attract such endogenous progenitors to the degenerated IVD and contribute to its repair [48, 49].

Presently, it is thought that discographies by the TAA are likely to induce acceleration of DDD or an increase in disc lesions [22, 50]. DDD is probably secondary to the iodine contrast agent (iCA) injection. Kim et al. recently showed that iCA is toxic to NP cells [51]. This toxicity is dependent, however, on the dose and on the nature of the agent $[51,52]$. Even though these in vitro results have to be verified under in vivo conditions, the DDD was thus probably induced by the injection of iCA rather than the AF puncture. Therefore, we strongly advocate avoiding the use of iCA when testing innovative cell therapies. In this in vivo study no effect of iCA injection was clearly observed on IVDs. However, the concentration of iodinated contrast agent used may not be sufficient to induce an IVD degeneration and the sample size was reduced. It would then be necessary to consider the effect of iCa on IVD through a longitudinal follow-up taking into account the dose injected, as in the study of Kim et al. [51].

\section{Conclusion}

To access the NP without inducing AF lesions, the TPA could be a suitable alternative to the conventional TAA dorsolateral NP access in the context of IVD regenerative medicine based on the injection of cells, biological factors or biomaterial into the NP. The TPA is, however, not without consequences, and the lack of healing of endplates at 7 months is a major limitation that may lead to accelerated DDD. Therefore, given to different trends observed in our work, we 
favour use of the TAA, which appears to be less invasive and does not induce any deleterious degenerative processes. This suggestion should be reassessed under specific conditions (a study of the efficacy of regenerative treatments). The TPA could nevertheless be indicated when injection into the IVD is preceded by a nucleotomy, and when the therapeutic objective is to replace the NP while keeping the AF intact. Thus, this study should be seen as a preliminary work that highlights the urgent need for additional studies in this regard, notably to more precisely assess the tissue consequences of the surgical routes used to target the NP.

\section{Publisher's Note}

Springer Nature remains neutral with regard to jurisdictional claims in published maps and institutional affiliations.

\section{Acknowledgements}

This study was supported by grants from the Société Française de Neurochirurgie, the Société Française de Chirurgie du Rachis, the Agence de la Biomédecine, the Institut National de la Santé et de la Recherche Médicale (INSERM), the Région des Pays de la Loire, ANR générique 2014 (REMEDIV project), the Fondation pour la Recherche Médicale FRM Bioingénierie (DBS20131128442), the Région des Pays de la Loire Research Program "Longévité Mobilité Autonomie" (LMA). The authors gratefully acknowledge the technical assistance that they received from the personnel of the CRIP (i.e. Patrice Roy, Christian Raphael, Stéphane Madec, Ingrid Leborgne, and Gildas Vaillant) and the SC3M platform (Service Commun de Microscopie électronique, Microcaractérisation et Morpho-histologie-imagerie fonctionnelle of the INSERM UMS016-SFR François Bonamy), as well as Sophie Domingues (Longdom publishing) for editing the manuscript.

Compilance with ethical standards

Conflict of interest All of the authors declare that they have no potential conflicts of interests.

Human and animal rights All applicable international, national, and/or institutional guidelines for the care and use of animals were adhered to. All procedures involving animals performed in this study were in accordance with the "3Rs" rule (Replacement, Reduction, and Refinement), the ethics standards of the institution and the practice at which the study was performed.

\section{References}


1. Luoma K, Riihimäki H, Luukkonen R et al (2000) Low back pain in relation to lumbar disc degeneration. Spine 25:487-492

2. Andersson GB (1999) Epidemiological features of chronic low-back pain. Lancet 354:581-585. https://doi.org/10.1016/S0140-6736(99)01312-4

3. Airaksinen O, Brox JI, Cedraschi C et al (2006) Chapter 4 European guidelines for the management of chronic nonspecific low back pain On behalf of the COST B13 Working Group on Guidelines for Chronic Low Back Pain. Eur Spine J 15: 192-300

4. Harrop JS, Youssef JA, Maltenfort M et al (2008) Lumbar adjacent segment degeneration and disease after arthrodesis and total disc arthroplasty. Spine 33:1701-1707. https://doi.org/10.1097/BRS.0b013e31817bb956

5. Colombier P, Camus A, Lescaudron L et al (2014) Intervertebral disc regeneration: a great challenge for tissue engineers. Trends Biotechnol 32:433-435. https://doi.org/10.1016/j.tibtech.2014.05.006

6. Henry N, Colombier P, Lescaudron L et al (2014) Regenerative medicine of the intervertebral disc: from pathophysiology to clinical application. Med Sci M/S 30:1091-1100. https://doi.org/10.1051/medsci/20143012012

7. Crevensten G, Walsh AJL, Ananthakrishnan D et al (2004) Intervertebral disc cell therapy for regeneration: mesenchymal stem cell implantation in rat intervertebral discs. Ann Biomed Eng 32:430-434

8. Sakai D, Mochida J, Iwashina T et al (2006) Regenerative effects of transplanting mesenchymal stem cells embedded in atelocollagen to the degenerated intervertebral disc. Biomaterials 27:335-345. https://doi.org/10.1016/j.biomaterials.2005.06.038

9. Orozco L, Soler R, Morera C et al (2011) Intervertebral disc repair by autologous mesenchymal bone marrow cells: a pilot study. Transplantation 92:822-828. https://doi.org/10.1097/TP.0b013e3182298a15

10. Pettine K, a., Murphy MB, Suzuki RK, Sand TT, (2015) Percutaneous injection of autologous bone marrow concentrate cells significantly reduces lumbar discogenic pain through 12 months. Stem Cells 33:146-156. https://doi.org/10.1002/stem.1845 
11. Alini M, Eisenstein SM, Ito K et al (2008) Are animal models useful for studying human disc disorders/degeneration? Eur Spine J 17:2-19. https://doi.org/10.1007/s00586-007-0414-y

\section{Hunter CJ, Matyas JR, Duncan NA (2004) Cytomorphology of} notochordal and chondrocytic cells from the nucleus pulposus: a species comparison. J Anat 205:357-362. https://doi.org/10.1111/j.00218782.2004.00352.x

13. Mageed M, Berner D, Jülke $H$ et al (2013) Is sheep lumbar spine a suitable alternative model for human spinal researches? Morphometrical comparison study. Lab Anim Res 29:183-189. https://doi.org/10.5625/lar.2013.29.4.183

14. Wilke HJ, Kettler A, Claes LE (1997) Are sheep spines a valid biomechanical model for human spines? Spine 22:2365-2374

15. Kapural L, Goyle A (2007) Imaging for provocative discography and minimally invasive percutaneous procedures for treatment of discogenic lower back pain. Tech Reg Anesth Pain Manag 11:73-80. https://doi.org/10.1053/j.trap.2007.02.013

16. Oehme D, Goldschlager T, Rosenfeld J et al (2012) Lateral surgical approach to lumbar intervertebral discs in an ovine model. Sci World J 2012:873726. https://doi.org/10.1100/2012/873726

17. Sobajima S, Kompel JF, Kim JS et al (2005) A slowly progressive and reproducible animal model of intervertebral disc degeneration characterized by MRI, X-ray, and histology. Spine 30:15-24

18. Michalek AJ, Funabashi KL, Iatridis JC (2010) Needle puncture injury of the rat intervertebral disc affects torsional and compressive biomechanics differently. Eur Spine J 19:2110-2116. https://doi.org/10.1007/s00586-010$1473-\mathrm{z}$

19. Li D, Yang H, Huang Y et al (2014) Lumbar intervertebral disc puncture under C-arm fluoroscopy: a new rat model of lumbar intervertebral disc degeneration. Exp Anim 63:227-234. https://doi.org/10.1538/expanim.63.227

20. Masuda K, Aota Y, Muehleman C et al (2004) A novel rabbit model of mild, reproducible disc degeneration by an anulus needle puncture : 
correlation between the degree of disc injury and radiological and histological appearances of disc degeneration. Spine 30:5-14

21. Wang JL, Tsai YC, Wang YH (2007) The leakage pathway and effect of needle gauge on degree of disc injury post anular puncture: a comparative study using aged human and adolescent porcine discs. Spine 32:1809-1815. https://doi.org/10.1097/BRS.0b013e31811ec282

22. Carragee EJ, Don AS, Hurwitz EL et al (2009) 2009 ISSLS Prize Winner: Does discography cause accelerated progression of degeneration changes in the lumbar disc: a ten-year matched cohort study. Spine 34:2338-2345. https://doi.org/10.1097/BRS.0b013e3181e234b5

23. Elliott DM, Yerramalli CS, Beckstein JC et al (2008) The effect of relative needle diameter in puncture and sham injection animal models of degeneration. Spine 33:588-596. https://doi.org/10.1097/BRS.0b013e318166e0a2

24. Kim KS, Yoon ST, Li J et al (2005) Disc degeneration in the rabbit: a biochemical and radiological comparison between four disc injury models. Spine 30:33-37

25. Vadalà G, Russo F, Pattappa G et al (2013) The transpedicular approach as an alternative route for intervertebral disc regeneration. Spine 38:E319324. https://doi.org/10.1097/BRS.0b013e318285bc4a

26. Vadalà G, De Strobel F, Bernardini M et al (2013) The transpedicular approach for the study of intervertebral disc regeneration strategies: in vivo characterization. Eur Spine J 22(Suppl 6):S972-978.

https://doi.org/10.1007/s00586-013-3007-y

27. Le Fournier L, Fusellier M, Halgand B et al (2017) The transpedicular surgical approach for the development of intervertebral disc targeting regenerative strategies in an ovine model. Eur Spine J 26:2072-2083. https://doi.org/10.1007/s00586-017-5199-z

28. Vinatier C, Gauthier O, Masson M et al (2008) Nasal chondrocytes and fibrin sealant for cartilage tissue engineering. J Biomed Mater Res Part A 89:176-185. https://doi.org/10.1002/jbm.a.31988

29. Boos N, Weissbach S, Rohrbach H et al (2002) Classification of agerelated changes in lumbar intervertebral discs: 2002 Volvo Award in basic 
science. Spine 27:2631-2644.

https://doi.org/10.1097/01.BRS.0000035304.27153.5B

30. Omlor GW, Lorenz S, Nerlich AG et al (2018) Disc cell therapy with bone-marrow-derived autologous mesenchymal stromal cells in a large porcine disc degeneration model. Eur Spine J 27:2639-2649. https://doi.org/10.1007/s00586-018-5728-4

31. Omlor GW, Fischer J, Kleinschmitt K et al (2014) Short-term follow-up of disc cell therapy in a porcine nucleotomy model with an albuminhyaluronan hydrogel: in vivo and in vitro results of metabolic disc cell activity and implant distribution. Eur Spine J 23:1837-1847. https://doi.org/10.1007/s00586-014-3314-y

32. Sheng SR, Wang XY, Xu HZ et al (2010) Anatomy of large animal spines and its comparison to the human spine: a systematic review. Eur Spine J 19:46-56. https://doi.org/10.1007/s00586-009-1192-5

33. Beckstein JC, Sen S, Schaer TP et al (2008) Comparison of animal discs used in disc research to human lumbar disc: axial compression mechanics and glycosaminoglycan content. Spine 33:E166-E173.

https://doi.org/10.1097/BRS.0b013e31824d911c

34. Omlor GW, Nerlich AG, Wilke $\mathrm{H}$ et al (2009) A new porcine in vivo animal model of disc degeneration: response of annulus fibrosus cells, chondrocyte-like nucleus pulposus cells, and notochordal nucleus pulposus cells to partial nucleotomy. Spine 34:2730-2739.

https://doi.org/10.1097/BRS.0b013e3181b723c9

35. Clouet J, Vinatier C, Merceron C et al (2009) The intervertebral disc: from pathophysiology to tissue engineering. Jt Bone Spine 76:614-618. https://doi.org/10.1016/j.jbspin.2009.07.002

36. Reitmaier S, Schmidt H, Ihler R et al (2013) Preliminary investigations on intradiscal pressures during daily activities: an in vivo study using the merino sheep. PLoS ONE 8:1-10.

https://doi.org/10.1371/journal.pone.0069610

37. Wognum S, Huyghe JM, Baaijens FPT (2006) Influence of osmotic pressure changes on the opening of existing cracks in 2 intervertebral disc models. Spine 31:1783-1788.

https://doi.org/10.1097/01.brs.0000227267.42924.bb 
38. Vadalà G, Sowa G, Hubert M et al (2012) Mesenchymal stem cells injection in degenerated intervertebral disc: cell leakage may induce osteophyte formation. J Tissue Eng Regen Med 6:348-355. https://doi.org/10.1002/term.433

39. Vadalà G, Russo F, De Strobel F et al (2018) Novel stepwise model of intervertebral disc degeneration with intact annulus fibrosus to test regeneration strategies. J Orthop Res 36:1-9.

https://doi.org/10.1002/jor.23905

40. May RD, Frauchiger DA, Albers CE et al (2018) Inhibitory effects of human primary intervertebral disc cells on human primary osteoblasts in a coculture system. Int J Mol Sci 19:4. https://doi.org/10.3390/ijms19041195

41. Urban JPG, Smith S, Fairbank JCT (2004) Nutrition of the intervertebral disc: spine. Spine 29:2700-2709

42. Benneker LM, Heini PF, Alini M et al (2005) Vertebral endplate marrow contact channel occlusions and intervertebral disc degeneration. Spine 30:167-173. https://doi.org/10.1097/01.brs.0000150833.93248.09

43. Määttä JH, MacGregor A, Karppinen J et al (2016) The relationship between Modic changes and intervertebral disc degeneration. BMC Musculoskelet Disord 17:371. https://doi.org/10.1186/s12891-016-1198-1

44. Haschtmann D, Stoyanov JV, Gédet P, Ferguson SJ (2008) Vertebral endplate trauma induces disc cell apoptosis and promotes organ degeneration in vitro. Eur Spine J 17:289-299. https://doi.org/10.1007/s00586-007-0509-5

45. Cinotti G, Della Rocca C, Romeo S et al (2005) Degenerative changes of porcine intervertebral disc induced by vertebral endplate injuries. Spine 30:174-180. https://doi.org/10.1097/01.brs.0000150530.48957.76

46. Sun W, Zhang K, Zhao C et al (2013) Quantitative T2 mapping to characterize the process of intervertebral disc degeneration in a rabbit model. BMC Musculoskelet Disord 14:357. https://doi.org/10.1186/1471-2474-14357

47. Clouet J, Fusellier M, Camus A, et al (2018) Intervertebral disc regeneration: from cell therapy to the development of novel bioinspired endogenous repair strategies. Adv Drug Deliv Rev. pii: S0169409X(18)30079-6 
48. Illien-Jünger S, Pattappa G, Peroglio M et al (2012) Homing of mesenchymal stem cells in induced degenerative intervertebral discs in a whole organ culture system. Spine 37:1865-1873. https://doi.org/10.1097/BRS.0b013e3182544a8a

49. Wangler S, Peroglio M, Menzel U et al (2019) Mesenchymal stem cell homing into intervertebral discs enhances the Tie2-positive progenitor cell population, prevents cell death, and induces a proliferative response. Spine 44:1613-1622. https://doi.org/10.1097/BRS.0000000000003150

50. Cuellar JM, Stauff MP, Herzog RJ et al (2016) Does provocative discography cause clinically important injury to the lumbar intervertebral disc? A 10-year matched cohort study. Spine J 16:273-280. https://doi.org/10.1016/j.spinee.2015.06.051

51. Kim KH, Park JY, Park HS et al (2015) Which iodinated contrast media is the least cytotoxic to human disc cells? Spine J. https://doi.org/10.1016/j.spinee.2015.01.015

52. Karaarslan N, Yilmaz I, Ozbek H et al (2019) Are radio-contrast agents commonly used in discography toxic to the intact intervertebral disc tissue cells? Basic Clin Pharmacol Toxicol 124:181-189. https://doi.org/10.1111/bcpt.13112 